\title{
Managed Care Best Practices The Road From Diagnosis to Recovery: Access to Appropriate Care
}

\author{
Michael Golinkoff, PhD, EMBA
}

\begin{abstract}
BACKGROUND: Depression is a painful, functionally impairing, and frequently recurring condition. A costly illness for employers, depression contributes to reduced functioning, decreased work productivity, and absenteeism. In addition, costs associated with comorbidities in people who have depression are higher when they are compared with the costs in people who do not have depression. In patients with either coronary or cerebral vascular disease, depression significantly increases the risk of cardiovascular morbidity and mortality.
\end{abstract}

OBJECTIVE: To highlight some of the challenges faced by primary care providers when they deliver mental health services and to provide an overview of an integrated behavioral health and medical benefit plan implemented as one solution to improve overall depression outcomes and reduce associated depression costs.

SUMMARY: Because of the high prevalence and significant impact of depression, the U.S. Preventive Services Task Force recommends screening adults for depression in clinical practices that have systems in place to ensure accurate diagnosis, effective treatment, and follow-up. However, depression appears to be only partially recognized and inadequately treated in primary care due somewhat to several challenges that primary care practitioners face when they manage a mental health diagnosis along with comorbid medical illnesses.

CONCLUSION: Integrating the medical and behavioral health benefits in a health plan allows for depression screening and the opportunity to direct patients to seek treatment when needed. Preliminary results of this effort in one managed care plan show reduced use of hospital services, days gained at work, and improved adherence to antidepressant therapy. A different component of this plan design includes patient care coaching as well as support of primary care's efforts by providing expert telephone consultation about treatment plans and allowing billing for adequate time spent with depressed patients.

J Manag Care Pharm. 2007;13(9)(suppl S-a):S23-S27

Copyright@ 2007, Academy of Managed Care Pharmacy. All rights reserved.

\section{Author}

MICHAEL GOLINKOFF, PHD, EMBA, is the chief clinical officer, Aetna Behavioral Health, King of Prussia, Pennsylvania.

AUTHOR CORRESPONDENCE: Michael Golinkoff, PhD, EMBA, Head, Clinical and Service Delivery, Aetna Behavioral Health, 1100 First Avenue, Suite 200, King of Prussia, PA 19406. Tel.: 610.783.4125; Fax: 860.975.1249; E-mail: golinkoffm@aetna.com $\square$ epression has a lifetime prevalence rate of $6.3 \%$ in men and about $12.6 \%$ in women. ${ }^{1}$ It is a tremendously costly illness for employers as it results in impaired functioning, decreased work productivity, and increased absenteeism. ${ }^{2}$ Among patients who suffer from concomitant depression, the costs for managing diabetes, hypertension, and ischemic heart disease approximately double. ${ }^{3}$ Depression is also associated with worse outcomes for cardiovascular disease. ${ }^{4}$ Partially in response to recognition of the significant prevalence and morbidity of depression, the U.S. Preventive Services Task Force recommends screening adults for depression in clinical practices that have systems in place to ensure accurate diagnosis, effective treatment, and follow-up. ${ }^{5}$ Although only about 25\% of patients who seek help for depression in the primary care setting receive adequate treatment, there is increasing evidence that practice-based quality improvement efforts for depressed patients can improve quality of care and depression-related outcomes. ${ }^{4,6-8}$

\section{Employer Costs}

Depression adversely affects functioning in all domains, but only recently has the magnitude of the impact on work productivity become clear. Productivity at work is an important businessrelated metric, and depression-specific costs to corporate America are beginning to be convincingly quantified. ${ }^{6}$ Depression substantially reduces an employee's capacity to work, which is partially explained by a depressed employee's lack of energy, insight, and creativity or motivation..$^{2,6,9,10}$ Stewart and colleagues ${ }^{2}$ calculated the cost of lost labor associated with depression in a survey of U.S. workers and estimated that depressed workers cost employers $\$ 44$ billion per year. Most (81\%) lost productive work time costs are invisible and are explained by reduced performance at work. Specifically, 5.6 hours per week were calculated as lost productive time experienced by all workers with depression of any severity, 8.4 hours per week by workers with major depression, and 1.5 hours per week for workers without depression.

Absenteeism is also an important business-related metric because employers spend an average of $1.9 \%$ of payroll expenditures on sick leave benefits. ${ }^{6}$ Major depression is a substantial but addressable contributor to absenteeism. In Stewart's analysis, $\$ 8.27$ billion of lost dollars was attributable to the cost of lost productive time because of absenteeism in workers with depression. ${ }^{2}$ In a separate study, improved depression management reduced absenteeism by $22.8 \%$ in all depressed workers and by $28.4 \%$ in consistently employed depressed workers, indicating gaps in care and opportunities for improvement. ${ }^{6}$

\section{Unemployment}

A study to determine the effect of depression on employment status showed that the presence of depression is linked with 
subsequent unemployment even after adjusting for confounding variables, including marital status, education, history of unemployment, current part-time employment, and cigarette smoking. ${ }^{9}$ Depressive symptoms were associated with a $60 \%$ increased adjusted odds of subsequent unemployment; $33 \%$ of participants with depressive symptoms and $21 \%$ of participants without depressive symptoms reported new unemployment during the 5 years after study initiation. ${ }^{9}$ Depression was also associated with subsequent reduction in family income. In one study that compared 1990 with 1991 annual incomes to onset of depressive symptoms, $17 \%$ of the participants who had depressive symptoms reported that their family incomes had decreased below $\$ 25,000$ within 5 years of developing depression compared with only $7 \%$ of participants without substantial depressive symptoms.

\section{Depression and Concomitant Medical IIIness}

Chronic medical illnesses and depression are often seen concomitantly. The presence of a chronic medical illness increases the likelihood of a concomitant depressive episode, and depression has been shown to adversely affect the course of coexisting medical illness, particularly for diabetes and cardiovascular conditions. ${ }^{11-13}$ Depression is associated with decreased glycemic control and increased micro- and macrovascular complications in patients with diabetes, and it also increases the risk of developing diabetes by about $23 \%{ }^{11,14}$ The mechanism of this effect is not understood but is hypothesized to include depressioninduced abnormalities in neuroendocrine and neurotransmitter function or decreased self-care behaviors. ${ }^{11,14}$

Considerable data from prospective studies with recognized indices of depression and objective outcome measures support depression as an independent cardiac risk factor. ${ }^{15}$ Many large-scale, well-controlled studies-in which initially healthy subjects were followed up prospectively-identified depression as a significant independent risk factor for both first myocardial infarction (MI) and cardiovascular mortality. ${ }^{15}$ Similarly, among individuals with established ischemic heart disease, depression has been found to be associated with an approximately 3- to 4-fold increase in the risk of subsequent cardiovascular morbidity and mortality. ${ }^{12}$ After an acute MI, depression is a risk factor for mortality independent of cardiac disease severity. ${ }^{13}$

\section{Depression Treatment and Medical Outcomes}

The treatment of depression and subsequent effect on existing medical illness is an area of opportunity for continued research. It has been shown that improving depression care in patients with arthritis not only improves depressive symptoms, but also improves pain symptoms, functional status, and quality of life. ${ }^{16}$ In patients with relatively well-controlled diabetes, depression interventions improved self-care and depression outcomes, but diabetes outcomes were not affected. ${ }^{11}$ The use of selective serotonin reuptake inhibitors (SSRIs) has been shown to be safe to use in patients with ischemic heart disease or coronary heart disease. ${ }^{16}$ But no large, randomized controlled trials to date have shown that treating depression in patients with cardiovascular disease improves outcomes in terms of costs, morbidity, and/ or mortality. The National Heart, Lung, and Blood Institute Working Group commented that there is a need for a multicenter, randomized, controlled trial to determine whether effective treatment for depression can reduce risk of cardiovascular disease events and mortality associated with depression in coronary heart disease patients. ${ }^{17}$ Unlike the general psychiatric literature that shows psychotherapy is as effective a treatment for depression as is pharmacotherapy, large randomized, controlled trials have not shown a positive impact of psychotherapy for treating depression in cardiovascular patients.

The next section highlights recent research investigating the safety and efficacy of antidepressants in heart disease. Although much research exists on the associations between depression and physical diseases, this discussion is limited to a presentation of 3 large trials of depression and heart disease.

\section{Sertraline Antidepressant Heart Attack Randomized Trial (SADHART)}

In line with increasing awareness of the links between depression and cardiovascular morbidity and mortality, a study was undertaken to evaluate the safety and efficacy of an SSRI, sertraline, in patients hospitalized post-MI or with unstable angina who had also been diagnosed with a major depressive disorder. $^{12}$ It was shown that sertraline is safe to use in a population with ischemic heart disease and does not impose cardiovascular risk. In patients who had severe depression or recurrent depression, sertraline provided greater improvement in depression symptoms than did placebo. But for mild depression or when the initial depressive episode occurred post-MI, sertraline showed no superior antidepressive effect compared with placebo.

\section{Cardiac Randomized Evaluation of Antidepressant and Psychotherapy Efficacy (CREATE)}

The CREATE study, ${ }^{18}$ a 12 -week trial involving 284 patients with coronary artery disease (CAD), compared efficacy, safety, and tolerability of weekly interpersonal psychotherapy; 20-40 mg of citalopram daily; or weekly standardized clinical management, alone or in combination, to treat major depression in CAD patients. Clinical management involved weekly group sessions with information about depression and medication use, reassurance, and encouragement for medication adherence. Interpersonal psychotherapy involved sessions addressing problems common in patients with CAD, including interpersonal conflicts, life transitions, grief, and loss.

The trial, a 2-by-2 factorial design, studied 4 groups: (1) interpersonal psychotherapy plus placebo, (2) interpersonal psychotherapy plus citalopram, (3) clinical management plus placebo, and (4) clinical management plus citalopram-permitting the 
evaluation of both interpersonal psychotherapy and citalopram. The researchers found that the depression responded to the SSRI and that there was a greater difference in depression response rate if there was a prior history of depression. They also found no advantage to adding psychotherapy to medical treatment of depression, although some editorials cautioned against dismissing the value of psychotherapy for depression in this population. ${ }^{19}$ Whether SSRIs reduce future cardiac events has not been firmly established.

\section{Enhancing Recovery In Coronary Heart Disease (ENRICHD)}

Recognizing the strong association between morbidity and mortality in patients post-MI who have low perceived social support and depression, Berkman et al. undertook a study to determine whether mortality and recurrent infarction are reduced by antidepressant treatment in this population. ${ }^{13}$ Patients $(\mathrm{N}=2,481)$ were randomized to receive either usual care or intervention therapy to address their depression. Intervention therapy began with cognitive behavior therapy in all patients. After 5 weeks, if patients continued to be severely depressed (defined by a Hamilton Depression Rating Scale [HDRS] rating $>24$ ) or had no response to therapy (defined as $<50 \%$ reduction from the initial Beck Depression Inventory score), cognitive behavior therapy was supplemented with an SSRI antidepressant, or, if not tolerated, an alternate antidepressant. All therapies were continued for 6 months after study initiation. The primary outcome measures were death or recurrent MI, while secondary outcomes included change in depression or perceived low social support scores at 6 months.

At 6 months, intervention decreased depression scores and improved social support more often than usual care. After an average follow-up of 29 months, across all patients there was no significant difference in event-free survival between usual care and psychosocial intervention. However, results from a post hoc analysis indicate that while cognitive therapy did not reduce mortality or recurrent MI, patients receiving SSRIs did have a $42 \%$ reduction in death or recurrent MI compared with depressed patients not on medication..$^{20}$ Among the rationale for this encouraging outcome: antiplatelet effects of SSRIs. These post hoc findings, although intriguing, should be interpreted cautiously, and the potential benefits of SSRIs on cardiac endpoints should be ascertained in a future study with random assignment to pharmacotherapy.

\section{Likelihood of Adequately Treated Depression}

Depression, one of the most costly and debilitating disorders, is inadequately identified and treated in many patients. Kessler and colleagues ${ }^{7}$ presented sobering data on the likelihood of medically treated and adequately treated depression. In the National Comorbidity Survey Replication of people who reported having major depressive disorder, $51.6 \%$ received health care treatment within the previous 12 months. Within this population, only $42 \%$ of the treatment they received was deemed to be minimally adequate ( $64.3 \%$ if they were treated by a mental health specialist and $41.3 \%$ if they were treated in a general medical setting). These findings demonstrate striking gaps between the use of existing effective depression treatments and most current practices, where depression identification and management fall short of the guidelines.

An analysis of 358 patients newly started on antidepressants in primary care clinics (within a staff model organization) showed that 195 (54.5\%) received doses recommended by the Agency for Health Care Policy and Research for 90 days or more. ${ }^{21}$ Although no significant differences in improvement of health-related quality of life outcomes during 6 months were observed, mean total medical costs over 6 months for patients taking the recommended levels of antidepressant treatment were $\$ 1,872 \pm \$ 140$ compared with $\$ 2,622 \pm \$ 413$ for patients taking less than recommended treatment $(P=0.032)$. The differences in total medical costs were attributable to significantly lower nonmental health-related inpatient costs in the recommended antidepressant treatment group ( $\$ 104$ vs. $\$ 785, P=0.004) .^{21}$

Another study showed that depression care and outcomes can be enhanced by supporting primary care practitioners (PCPs) in the treatment and management of their depressed patients. This care model includes education on best practices, nurse clinicians who provide regularly scheduled patient-directed care management, and engagement of patients to be active in their own treatment. ${ }^{22}$ For patients beginning new treatment for depression, 2 years of enhanced care significantly increased the number of days free of depression impairment when compared with usual care, and the incremental cost-effectiveness ratio for enhanced care ranged from $\$ 9,592$ to $\$ 14,306$ per qualityadjusted life-year. The number of incremental days free of depression impairment increased from 23 days in the first year to 36.4 days in the second year $(P<0.001)$, while incremental health plan costs decreased significantly from $\$ 568$ in the first year to $-\$ 12$ in the second year $(P<0.001){ }^{22}$

\section{Aetna Behavioral Health Programs}

The next section provides an overview of the efforts of one large national payer, Aetna, to focus on plan design strategies aimed at improving depression and comorbid outcomes. By integrating medical and behavioral health services and program offerings, Aetna coordinates members' care to include treatment coverage of the mental health aspects as well as any chronic medical illness. For example, various programs outside of behavioral health exist within Aetna, and all of them screen for depression every time there is verbal contact with a member and the member consents to the screening. About 6,000-8,000 members are screened for depression every month. When patients screen positive, they are alerted with recommendations about using their mental health benefit and asked if a care coach can contact them by telephone. In this way, they are referred to the 
behavioral health program for further assessment and intervention. If warranted, a care management plan that involves care coordination within Aetna and the patient's physician is developed for the member.

A similar process is used for targeted member interventions. Members who appear to have depression are identified by pharmacy and medical claims and stratified on the basis of mild, moderate, or severe medical issues based on predictive models developed by Aetna to determine the level of intervention. Program interventions include an outreach, assessment, and enrollment; payer coordination of care between the medical and behavioral health care providers; patient telephonic coaching by behavioral health specialists and active case management for more severe depression; or educational information mailings for relatively mild depression. Because of a very high comorbidity between alcohol use and depression, screening for alcohol use is also a component. A separate program uses similar processes to identify and provide care for patients hospitalized with behavioral health issues, such as bipolar disorder or major depression. There are also less intense behavioral health offerings, such as employee assistance programs for relatively mild depressive symptoms for which counseling about workplace, work/life, legal, or financial issues is provided.

Initial outcomes from this fairly new program show minimal improvement in physical health, but improvements in mental health measures seem to be supported by observed reductions in HDRS scores, improvement in energy level, reduction in work limitations, and reduction in social limitations (statistical analysis not completed). One of the programs for more severely affected members showed that members gained 7.7 work days per month. A very preliminary assessment of the cost profile indicates reduced emergency room use, inpatient length of stay, outpatient visits, and behavioral health inpatient stays with associated reductions in costs in these areas. Antidepressant adherence improved by $10 \%$.

\section{Depression in the Primary Care Setting}

As U.S. medical care has evolved, PCPs have been expected to recognize and treat mental health problems. ${ }^{23}$ Primary care clinicians are expected to identify signs of possible mental health problems, incorporate those observations into differential diagnoses, and decide which problems to treat or monitor and which to send for consultation or referral. ${ }^{23}$ In addition, a patient may or may not have a primary diagnosis and mood disorders frequently associated with comorbidities, such as anxiety or substance abuse. Thus, PCPs face challenges in meeting a patient's mental health needs effectively, and the decisions that they make have important financial and health consequences, especially regarding care for mood disorders.

The Aetna "Depression in the Primary Care Program" was implemented partly because it is recognized that most people who have depression choose to be treated in the primary care setting, and it is understood that PCPs have unique challenges in not only identifying and treating the depressed patient but also in billing for the services provided. In general, a behavioral health benefit does not typically cover the treatment of psychiatric disorders offered by a nonmental health professional. Therefore, reimbursement for PCPs treating depression is problematic in many payer situations. In addition, many medical visit codes are billable for no more than 15 minutes, and depressed patients often require more time.

In the "Depression in the Primary Care Program," PCPs receive training in person or via the Internet on identification, stratification, and treatment of depression. Unique to this program is that the PCP is allowed to bill the medical benefit for behavior health services; additionally, the PCP can bill for the actual amount of time needed to treat the patient with depression rather than the typical one 15-minute billing unit.

Patient members enroll in the program via the Internet and complete a Web-based patient screening and assessment tool, which screens for most of the major psychiatric conditions and comorbid anxiety or substance abuse disorders. Patients link their PCPs to their assessments, which allows their PCPs to access the results via a secure Web site. When the assessments are complete, their providers receive an e-mail alert. In addition, an Aetna care clinician will review the data to verify a diagnosis of depression and to determine whether a psychiatric comorbidity is possible. If needed, the care clinician is able to consult with the PCPs by telephone to discuss optimizing patient treatments. Members can access the site again for reassessments after treatment, and the program provides depression-progress tracking tools. Similar to the alert received after the first assessment, another e-mail alerting PCPs to the updated information is sent.

Preliminary results of 41 enrolled members indicate improvement in depression severity scores and show that those with minimal symptoms have minimal improvement and those with more severe depression show more improvement in disease severity. This outreach program may also be particularly effective for the depressed elderly population. Care management programs for the elderly population may be even more effective than they are for the younger population. In the elderly population, the program provides some social contact for a group of people in isolation and provides education where there is a general lack of understanding. This program also helps mitigate a more complex issue in this group by coordinating and integrating medical care, among often greater numbers of treating practitioners.

Initial results are showing the program to be going in the right direction and as more patients enroll in the program, results continue to be collected. A few enhancements have been made, most notably, communicating this program to the clinic office staff, who, in turn, inform patients about the program and encourage them to enroll. 


\section{Summary}

In a managed care setting, benefit design may improve depression-related outcomes and reduce overall costs related to depression. Integrating the medical and behavioral health benefits in a health plan allows for screening of depression and directing of patients to seek treatment when they need it. Preliminary results of this effort in one managed care plan show reduced use of hospital services, days gained at work, and improved adherence to antidepressant therapy. In a different component of this plan design, patient care coaching, along with support of primary care's efforts by providing expert telephonic consultation on treatment plans and allowing billing for adequate time spent with depressed patients, produced initial results indicating improvement in depression severity ratings. In conclusion, further study of this approach is needed to fully realize whether there are benefits of improved work productivity or any cost reductions related to better depression care.

\section{REFERENCES}

1. Riolo SA, Nguyen TA, Greden JF, King CA. Prevalence of depression by race/ethnicity: findings from the National Health and Nutrition Examination Survey III. Am J Public Health. 2005;95:998-1000.

2. Stewart W, Ricci JA, Chee E, Hahn S, Morganstein D. Cost of lost productive work time among U.S. workers with depression. JAMA. 2003;289:3135-44

3. Escobar JI. The psychiatrist's view of depression. Manag Care. 2006; 15(suppl 9). Available at: www.managedcaremag.com/supplements/ 0610_mood/MC_0610_MOOD.pdf.

4. Koike AK, Unutzer J, Wells KB. Improving care for depression in patients with comorbid medical illness. Am J Psychiatry. 2002;159:1738-45.

5. U.S. Preventive Services Task Force. Screening for depression in adults: summary of the evidence. Ann Intern Med. 2002;136:765-76.

6. Rost K, Smith JL, Dickinson M. The effect of improving primary care depression management on employee absenteeism and productivity. A randomized trial. Med Care. 2004:42:1202-10.

7. Kessler RC, Berglund P, Demler O, et al. The epidemiology of major depressive disorder: results from the National Comorbidity Survey Replication (NCS-R). JAMA. 2003;289:3095-105.

8. Katon WJ, Rutter C, Simon G, et al. The association of comorbid depression with mortality in patients with type 2 diabetes. Diabetes Care. 2005;28:2668-72.

9. Whooley MA, Keife CI, Chesney M, Markovitz JH, Matthews K, Hulley SB. Depressive symptoms, unemployment, and loss of income. Arch Intern Med. 2002;162:2614-20.
10. Kessler RC, Greenberg PE, Mickelson KD, Meneades LM, Wang PS. The effects of chronic medical conditions on work loss and work cutback. J Occup Environ Med. 2001;43:218-25.

11. Williams JW Jr., Katon W, Lin E, et al. The effectiveness of depression care management on diabetes-related outcomes in older patients. Ann Intern Med. 2004;140:1015-24.

12. Glassman AH, O'Connor CM, Califf RM, et al. for the Sertraline Antidepressant Heart Attack Randomized Trial (SADHART) group. Sertraline treatment of major depression in patients with acute MI or unstable angina. JAMA. 2002;288:701-09.

13. Berkman LF, Blumenthal J, Burg M, et al. for the Enhancing Recovery In Coronary Heart Disease Patients investigators (ENRICHD). Effects of treating depression and low perceived social support on clinical events after myocardial infarction: the Enhancing Recovery In Coronary Heart Disease Patients (ENRICHD) randomized trial. JAMA. 2003;289:3106-16.

14. Brown LC, Majumdar SR, Newman SC, Johnson JA. History of depression increases risk of type 2 diabetes in younger adults. Diabetes Care. 2005;28:1063-67.

15. Frasure-Smith N, Lespérance F. Reflections on depression as a cardiac risk factor. Psychosom Med. 2005;67:S19-S25.

16. Lin EH, Katon W, Von Korff M, et al. for the Improving MoodPromoting Access to Collaborative Treatment (IMPACT) investigators. Effect of improving depression care on pain and functional outcomes among older adults with arthritis: a randomized controlled trial. JAMA 2003;290:2428-29.

17. Davidson KW, Kupfer DJ, Bigger JT, et al. Assessment and treatment of depression in patients with cardiovascular disease: National Heart, Lung, and Blood Institute Working Group report. Psychosom Med. 2006;68:645-50.

18. Lespérance F, Frasure-Smith N, Koszycki D, et al. for the CREATE investigators. Effects of citalopram and interpersonal psychotherapy on depression in patients with coronary artery disease: The Canadian Cardiac Randomized Evaluation of Antidepressant and Psychotherapy Efficacy (CREATE) trial. JAMA. 2007;297:367-79.

19. Dornelas EA, Burg MM. Treatment of depression in patients with coronary artery disease. JAMA. 2007;297:1879-80.

20. Taylor CB, Youngblood ME, Catellier D, et al. for the ENRICHD investigators. Effects of antidepressant medication on morbidity and mortality in depressed patients after myocardial infarction. Arch Gen Psych. 2005; 62:792-98

21. Revicki D, Simon G, Chan K, Katon W, Heilgenstein J. Depression, health-related quality of life, and medical cost outcomes of receiving recommended levels of antidepressant treatment. J Fam Pract. 1998; 47:446-52

22. Rost K, Pyne JM, Dickinson LM, LoSasso AT. Cost-effectiveness of enhancing primary care depression management on an ongoing basis. Ann Fam Med. 2005;3:7-14.

23. Callahan EJ, Bertakis KD, Azari R, Robbins JA, Helms J, Leigh JP. Association of higher costs with symptoms and diagnosis of depression J Fam Pract. 2002;51:540-44. 